Capítulo V

\title{
Familia y discapacidad: calidad de vida y apoyo social percibido
}

Floralinda García Puello • Estela Crissién Quiroz 


\section{CONCEPTUALIZACIONES EN TORNO A LA FAMILIA}

La familia, en esencia, posee un carácter de institución continente y de unidad fundante de la sociedad; es considerada una organización cuyos miembros están unidos por relaciones de alianzas y de consanguinidad, los cuales se encuentran expuestos, por un lado, a la interacción con el medio externo, y por otro, a las exigencias propias del desarrollo de su medio interno, produciendo influencias de diverso orden e intensidad, aunque inevitablemente relacionadas (1).

La familia también es considerada una institución social y cultural dinámica, influenciada por diversas áreas de la sociedad (económica, social, cultural y política); que determinan en gran parte su desarrollo y movimiento (1). Por tanto, se considera que esta sobrepasa los vínculos de sangre o jurídicos e integra a quienes hagan parte del núcleo afectivo central. La familia puede definirse desde diferentes perspectivas de acuerdo con el tipo de convivencia que tengan sus miembros, el intercambio de bienes económicos, la intimidad, la protección, la educación y el desarrollo personal de los mismos. Como consecuencia de esto, existen diversos tipos de familia, los más comunes son la familia nuclear, integrada por padre, madre e hijos y familia monoparental, caracterizada por la presencia de uno de los progenitores y los hijos, los cuales normalmente son dependientes del padre o la madre (2).

No obstante, independientemente de esta clasificación, si la familia es considerada un sistema, y ejerce sus funciones básicas adecuadamente, se convierte en una fuente de salud social que se nutre a sí misma y a todo lo que le rodea. Al concebir a la familia como un sistema, se entiende que la interacción de sus miembros tiene una jerarquía, unos límites y unas funciones que se autorregulan internamente y que están en permanente relación con su contexto (3). Esta definición y características de la familia, surgen prin- 
cipalmente en la etapa moderna, luego de un período de transición que evidencia el paso de un modelo de familia nuclear biparental, donde la figura masculina primaba como jefe, a una amplia gama de tipologías familiares que coexisten con diferentes modalidades de funcionalidad, unidas a procesos de transformación permanentes a lo largo del ciclo vital (4).

Ambas formas de funcionalidad familiar, las tradicionales nucleares, las modernas y postmodernas, han cumplido y cumplen con unas funciones internas y externas que impactan directamente sobre la vida de las personas que las integran; y si bien, en algunas sociedades, sobre todo occidentales, sigue primando el tipo de familia tradicional nuclear, donde la autoridad es ejercida estrictamente bajo el mando del varón, con una división sexual del trabajo, y una premisa de jerarquía de género y edad, no es esta una condición necesaria para lograr la estabilidad de la organización familiar, ni el aumento de bienestar que esta necesita para funcionar (4). Incluso, desde el punto de vista fenomenológico, resulta más trascendental la funcionalidad que la familia pueda desarrollar, en beneficio de sus miembros, independientemente de su tipo de organización, que la consideración de la familia nuclear como "la célula básica de la sociedad", necesaria para la construcción de un tejido social (4).

Por tanto, al hablar de familia, en relación con el fenómeno de la discapacidad, debemos aliarnos a un concepto amplio y flexible, reconociéndola desde toda clase de vínculos, más allá del parentesco o de la tutela legal (3). Esta concepción hace parte del enfoque sistémico, en el cual la familia es definida como un sistema, en el que cada uno de sus miembros está regulado por las relaciones que tienen entre sí todos los elementos del sistema familiar; es decir, existe una interrelación entre todos los individuos que conforman la familia, de tal forma que un cambio dentro o fuera del núcleo familiar podría afectar a cualquiera de los miembros que la integran (5). 
Desde este enfoque, la familia se convierte en la primera y más importante red de apoyo para las personas que la conforman, porque es la que sostiene y amortigua las tensiones de la vida.

Desde el enfoque de derechos humanos, se reafirma el papel primario y fundamental que tiene la familia en la sociedad y, por tanto, la protección que requiere y merece como tal (2); más aún, tratándose de familias que presentan algún miembro en situación de discapacidad. Este tipo de familias, su estructura y funcionalidad, afectará en gran medida el desarrollo de este miembro, tanto en su vida personal dentro de la familia, en el progreso de su personalidad, como en su relación con el medio en el que cotidianamente se mueve. Es aquí donde cobra gran importancia el apoyo que el Estado proporcione a la familia, apoyo expresado a través del suministro de ambientes accesibles y equiparación de oportunidades en todas las áreas que componen la vida humana: salud, educación, trabajo, protección social y seguridad (6).

De forma natural, la familia está expuesta permanentemente a cuestionamientos, inquietudes y necesidad de respuestas para el mantenimiento de su desarrollo y permanencia en la sociedad; esto es coherente si se tiene en cuenta la dinámica a la que está expuesta, la diversidad de roles y los retos socioculturales y económicos impuestos por el contexto vivencial; sin embargo, toda esta carga de necesidades se ve aumentada ante la presencia de uno de sus miembros con alguna discapacidad, y es aquí donde se hace compleja la garantía de la funcionalidad familiar de tal forma que esta perciba claramente el goce efectivo de sus derechos en un contexto de equidad (7); un contexto donde encuentre recursos suficientes para mantener el cuidado de sí misma y de cada uno de sus miembros.

No obstante, la realidad social no es muy grata ante esta necesidad; y esto se ve reflejado en la variedad de papeles que la familia debe cumplir para 
poder subsistir, más aún con uno de sus miembros con alguna discapacidad. Aspectos como la necesidad de trabajo por parte de las mujeres, las cuales siguen siendo las principales responsables del cuidado de la persona con discapacidad con la consecuente reducción de tiempo para ejercer estos cuidados, son un ejemplo claro de esta realidad (8).

Debemos reconocer, que independientemente del carácter organizativo familiar, de su tipología y composición estructural, la familia juega un papel trascendental en el desarrollo de todo ser humano, un papel que ejerce como agente socializador, protector y transmisor de valores y normas que van a coadyuvar de forma importante en la construcción de la identidad personal. Este entramado de significancia, adquiere más relevancia cuando el objetivo de análisis es la discapacidad, puesto que, ante este panorama, la familia se constituye en la unidad estructural y primera red de apoyo para la articulación de la persona con discapacidad a la realidad social.

Por tanto, resulta fundamental reconocer las problemáticas y posibles soluciones que presentan las familias, y en especial las que contienen uno de sus miembros con algún tipo de discapacidad; de tal forma, que toda la planeación de intervenciones que se puedan desarrollar desde diferentes fuentes de tipo gubernamental o no gubernamental, estén fundamentadas en el conocimiento profundo de su dinámica funcional, bajo el prisma de los derechos y equiparación de oportunidades (9).

\section{FAMILIAS CON PERSONAS EN SITUACIÓN DE DISCAPACIDAD}

Hoy se reconoce que la discapacidad es un fenómeno de gran importancia y que genera grandes retos, no solo para la salud pública mundial, sino también para todas las áreas que componen la sociedad en general. 
Para comprender mejor esto, se debe reconocer que las conceptualizaciones en torno a la discapacidad han evolucionado a lo largo del tiempo, pasando de ser concebida como un proceso netamente biológico e individualizado, donde predominaban las consideraciones clínicas y patológicas a ser comprendida como un proceso biopsicosocial, el cual impera en la sociedad actual (9) y evoluciona a partir de la interacción e interrelación del propio individuo que presenta la discapacidad con una serie de barreras contextuales de tipo actitudinal y del espacio físico que son las que en última instancia le bloquean las posibilidades de participación social de una manera plena y efectiva en igualdad de condiciones (7), realidad que se ve reflejada desde la interacción de la persona con discapacidad en el entorno familiar, considerado el primer contacto fundamental para el logro de su desarrollo y bienestar.

De acuerdo con esto, es importante reconocer que la presencia de una persona con discapacidad en el ambiente familiar, generará grandes cambios tanto en la estructura como en la dinámica de la funcionalidad familiar, la cual, dependiendo de su propia capacidad de respuesta, y presencia de fortalezas y debilidades podrá salir a flote de las dificultades que esto pueda generar. Ante esta situación, resulta de gran importancia, sensibilizar, informar, y acompañar a la familia ante un proceso que resultará difícil y complejo, pero que será real y necesario enfrentar (7).

Para la familia, la existencia de una enfermedad crónica severa o de una condición de discapacidad en uno de sus miembros, se ha asociado con un aumento del estrés familiar, disminución de la salud y aumento de la carga financiera. Los recursos psicológicos y materiales con los que esta cuenta para hacer frente a estas situaciones, varían de acuerdo con el tipo de familia y con la capacidad que tenga para funcionar eficazmente en medio de la adversidad (10). 
Ahora, la mayoría de los cuidados proporcionados a las personas con discapacidad provienen directamente de sus familias, particularmente, de los padres cuando es el hijo quien presenta la discapacidad. Tener un miembro en la familia con alguna condición de discapacidad, puede generar tensiones y estrés y, por ende, conflictos, separaciones, o por el contrario podría fortalecerse; esto dependerá en última instancia de factores como la comunicación, el bienestar y el apoyo social, que se vivan y perciban en el sistema familiar (5).

Existe un aspecto bien claro y es que todo grupo familiar, atraviesa por distintas etapas, conflictos, y estados de crisis que le permiten desarrollarse, reconocerse y consolidar sus lazos (1), el poseer uno de los miembros de la familia con alguna discapacidad hace parte de estos procesos, y en especial, este acontecimiento se convierte en trascendental porque llega a modificar en gran medida toda la dinámica familiar, las creencias, conceptos e ideales que el resto de los miembros tenían para con esta persona y en especial los padres. Así mismo, la presencia de la discapacidad en una familia, generará inevitablemente alteraciones de su equilibrio, no solo por el componente biológico del integrante, sino también por todas las repercusiones económicas y sociales que este fenómeno conlleva. Por tanto, la capacidad de respuesta y percepción positiva de este suceso al interior de la familia, garantiza en gran medida el desarrollo y evolución del integrante que presenta la discapacidad. A su vez, la capacidad de respuesta que la familia tenga frente a esta realidad, dependerá de múltiples factores dentro de los cuáles priman los caracteres, estados de ánimo, nivel cultural, el pronóstico y la gravedad de la situación de discapacidad (11), así como también el sistema de creencias de la familia, la etapa del ciclo vital familiar, el estigma social de la discapacidad y la propia experiencia que la familia pueda tener con una condición de discapacidad (8). 
Por otro lado, es importante tener en cuenta que la mayoría de las veces, casi ninguna familia está preparada para hacerle frente a la situación que encierra la presencia de uno de sus miembros con una discapacidad; esto es comprensible si se tiene en cuenta la diversidad de recursos necesarios para adaptarse a esta nueva situación y poder salir a flote (8).

Las alteraciones de la funcionalidad familiar pueden clasificarse de forma sintética en estructurales, de impacto emocional y de procesos. En las primeras, lo que principalmente se ve afectado son los roles y funciones de la familia; estos se modifican considerablemente hasta que se manifiesta claramente el rol del cuidador primario, el cual asume en su totalidad el cuidado y protección de la persona con discapacidad. Esta situación va generando progresivamente exclusiones al interior de la familia, dada principalmente por la relación tan estrecha y sobreprotección que llega a desarrollarse entre el cuidador y la persona con discapacidad. Frecuentemente, el desarrollo de este vínculo tan estrecho puede provocar resentimiento por otro integrante de la familia, desencadenando en el peor de los casos, sintomatología física y psicológica que complejiza aún más el cuadro familiar (8).

En relación con el componente emocional, la presencia de discapacidad en la familia genera una pluralidad de sentimientos y emociones: angustia, dolor, depresión, agresividad, culpabilidad y temor son algunos ejemplos de ellos. Así mismo, es muy frecuente que se presente en algunos de los miembros de la familia un sentimiento ambivalente, entre lo que verdaderamente están sintiendo y experimentando a raíz de la realidad de la situación, y lo que socialmente se espera que experimenten. De igual forma, la persona que ha asumido el rol de cuidador en la familia, puede experimentar sentimientos encontrados al sentir la necesidad natural de ayudar a la persona que presenta la discapacidad y al mismo tiempo sentir que esa responsabilidad puede convertirse en una carga difícil de soportar (8). 
Cuando una familia muestra características de aislamiento, con ausencia de redes familiares y si los lazos afectivos entre sus miembros son débiles y la comunicación no es asertiva, lo más probable es que la presencia de una persona con discapacidad, imponga nuevos o mayores retos que se sumarían a las deficiencias ya existentes (1). Esto requiere del suministro de servicios especiales temporales o permanentes dirigidos a las familias de las personas con discapacidad, para minimizar los obstáculos que puedan oponerse en el proceso de atención y cuidado de estas personas (2) y para lograr el mantenimiento de la calidad de vida familiar, la cual resulta fundamental si se tiene en cuenta que puede ser asumida como un indicador de éxito de los programas e iniciativas de políticas encaminadas a la prevención e intervención con esta población (12).

Diversos estudios han corroborado que cuando existe una guía u orientación para las familias de personas con discapacidad, en los casos de niños o niñas, esto les permite construir sus propios procesos de crecimiento familiar y de desarrollo de la independencia. Por tanto, se recomienda crear propuestas de apoyo para padres y madres, con el propósito de promover respuestas específicas a las necesidades que surgen en los diferentes períodos del ciclo de vida. Apoyo que se concibe como un trabajo interdisciplinario, integral y especializado. En el primero, contando con especialistas de diferentes áreas como lenguaje, terapia física, educación, entre otros; en el caso de trabajo integral, se considera importante el propiciar las dimensiones de desarrollo de la persona y la realidad familiar en la que vive el individuo con discapacidad y por último y no menos importante, el trabajo especializado de equipos profesionales que faciliten procesos de independencia que se transfieran, desde el hogar, hacia la escuela y la comunidad (12). Estos aspectos favorecerían la inclusión en la sociedad de las personas con discapacidad y sus familias, y permitiría disminuir los procesos de restricción en su participación social. 
Por otro lado, tomando como referencia la concepción que ofrece la Convención de las Naciones Unidas sobre la Discapacidad, donde resalta que esta se relaciona más que con una condición de salud específica, con un sinnúmero de barreras que le impiden al individuo con discapacidad participar de su contexto, resulta indispensable promover espacios sociales inclusivos que permitan que estas barreras sean eliminadas o disminuidas. Uno de estos espacios es precisamente el contexto familiar en el que se desenvuelven los individuos. La eliminación o disminución de las barreras sociales a las que se ven enfrentadas las personas con discapacidad deben iniciar desde su núcleo familiar puesto que es la familia el primer ente social que marcará muy particularmente el desarrollo social de la persona. Mientras mejor estructurados estén los lazos familiares y las interrelaciones interpersonales que en ella se presenten en aspectos emocionales, cooperativos, de comunicación y resolución de conflictos, el pronóstico de adaptación a las nuevas demandas que surgen a partir de la discapacidad serán mejores para los sujetos que la conforman (13).

Por otro lado, a partir de los aportes realizados por el enfoque social de la discapacidad, la perspectiva de la educación sobre la familia se ha ido transformando, y se ha iniciado un proceso de concienciación sobre el rol fundamental que esta tiene en el desarrollo del individuo. Hoy, es bien reconocido que es en la familia donde se suplen las necesidades sociales de los miembros que la conforman; por tanto, la familia debe ser identificada como un elemento clave para el desarrollo del individuo en todas sus dimensiones. Cuando se reconoce esto, se evidencia que la ruta más apropiada para lograr la participación activa de las personas con discapacidad en la sociedad, es a través de la intervención de la familia, dado que es esta la que soporta cualquier proceso personal o social de las personas, especialmente, cuando uno de sus miembros posee una condición de discapacidad (14). 


\section{CALIDAD DE VIDA EN FAMILIAS DE PERSONAS CON DISCAPACIDAD}

El concepto de calidad de vida en las personas con discapacidad y sus familias, ha cobrado cada vez más importancia, por lo cual se está avanzando en su medición, como primer y fundamental paso para determinar los apoyos requeridos para que la persona con discapacidad y su familia logren una mejor adaptación y desarrollo en sus contextos (12). Hablar de calidad de vida familiar en personas con discapacidad constituye un importante avance en los procesos de investigación sobre familia y discapacidad porque permite establecer claramente sus prioridades; además, amplía la perspectiva de orientación hacia la misma, resaltando los puntos fuertes de su funcionalidad y dándole confianza para desarrollar sus potencialidades y sus capacidades.

Desde esta concepción, se comprende la familia como una unidad de apoyo, coordinada y entrelazada con el equipo de profesionales de los servicios de atención para la persona con discapacidad y con otras familias, en la búsqueda de logros que respondan a sus necesidades reales, a sus expectativas, y al alcance de metas trascendentales para sus vidas $(5,6)$. Esto resulta fundamental si se reconoce que es la familia el sistema directo en el cual se adquieren las primeras interacciones humanas y por tanto es el sistema de entrada hacia el individuo con discapacidad (12).

La calidad de vida familiar es un concepto multifacético que surge con el objetivo fundamental de describir y comprender los factores determinantes de la calidad de vida y funcionalidad familiar, para tomarlos como referencia y línea de base en la construcción de políticas, planes, programas y servicios de intervención integrales encaminados a mejorar las condiciones de vida de todo el contexto familiar. El logro de estos objetivos serán los indicadores de la eficacia y la calidad de los servicios que se ofrecen e im- 
plementan en torno a la familia, los cuales se verán reflejados en mejores condiciones de vida para la persona con discapacidad (15).

A partir de los años 80 y 90, las investigaciones e intervenciones en las familias de personas con discapacidad comienzan a jugar un papel trascendental; sus necesidades en general, las de los padres y hermanos, de la comunidad y su relación con esta. Se profundizó en el análisis del papel que debe cumplir cada uno de los miembros de una familia con hijos o con alguno de sus miembros con discapacidad; se realizaron estudios sobre el estrés que maneja cada familia, aportando algunos estudios información útil para entender y afrontar los problemas que algunas familias tienen. Sin embargo, aún falta mucho por investigar para entender la realidad compleja que tienen estas familias. Esto, debido principalmente a que hay tantas familias como individuos, tan distintas como las personas, y con problemas tan diferentes por intervenir. Así mismo, hay muchos tipos de discapacidades y diferentes niveles de complejidad, los cuales afectarán en mayor o menor grado la estabilidad de la familia. En este sentido, los recursos con los que cuentan las familias pueden ser muy variados, al igual que los de la comunidad en la que viven (14).

Hablar de calidad de vida en las familias que tienen uno de sus miembros con discapacidad es analizar el impacto que la discapacidad puede tener sobre la calidad de vida de la familia, así como también, analizar el papel que las familias deben desempeñar para que la calidad de vida de las personas con discapacidad sea lo mejor posible. Este es por lo tanto, un enfoque centrado en la familia, donde no tiene mucha importancia el enfoque exclusivamente clínico, que procura intervenir la discapacidad, sino un enfoque que busca la colaboración de la familia como el apoyo principal para la persona con discapacidad y sus posibilidades de acceso a la sociedad (14).

El modelo de calidad de vida centrado en la familia la estimula a tomar la 
iniciativa en el establecimiento de sus prioridades; abandona la perspectiva patológica y adopta una orientación desde los puntos fuertes de la familia, dándole confianza para desarrollar sus potencialidades y sus capacidades. Así mismo, este enfoque considera a la familia entera como una unidad de apoyo, que trabaja en equipo con los profesionales de los servicios de atención para la persona con discapacidad, y con otras familias en la búsqueda de logros que respondan a sus necesidades reales y a sus expectativas (16).

En coherencia con lo anterior, se reconoce, por tanto, la necesidad de evaluar la variable de calidad de vida desde una perspectiva integral, en la que se incluyan indicadores objetivos o sociales y subjetivos o medidas de bienestar. Esta concepción de calidad de vida en general constituye el marco de referencia para el concepto de calidad de vida familiar y, por ende, de calidad de vida en familias de personas con discapacidad $(14,15)$.

En países como Estados Unidos y Canadá, se ha propuesto que la calidad de vida de las familias de personas con discapacidad debe ser asumida como un indicador de éxito de los programas e iniciativas de políticas encaminadas a la prevención e intervención con esta población; así mismo, equipos de investigadores en diferentes partes del mundo realizan cada vez mayores esfuerzos para avanzar en la comprensión y en la medición del constructo "calidad de vida" en el ámbito de la discapacidad. Igualmente, señalan la relación entre la satisfacción con la calidad de vida familiar con variables como tipo de discapacidad, edad, género, tipo de familia, estatus socioeconómico. Algunos resultados de investigaciones han encontrado que el factor en que las familias presentan mayor insatisfacción es en el de apoyo a la persona con discapacidad y los indicadores de apoyo para conseguir beneficios del Gobierno y otras entidades locales (13). Este es un aspecto a tener en cuenta para el desarrollo de intervenciones enfocadas hacia las personas con discapacidad, si se pretende impactar sobre su de- 
sarrollo humano expresado en la inclusión social en un marco de equiparación de oportunidades en la sociedad.

Uno de los aspectos que pueden afectar la calidad de vida de la familia es el relacionado con los cuidados que deben proporcionársele al integrante que presenta la discapacidad, como consecuencia de los cuales pueden experimentarse cambios en la vida cotidiana, transformación de roles y exceso de responsabilidades familiares (17). Son diversas las investigaciones que reportan lo aquí señalado; un ejemplo es el estudio de sobrecarga en cuidadores familiares realizado en España, el cual muestra una relación positiva entre las dimensiones de la carga familiar, las características del paciente, del cuidador, los síntomas de la enfermedad y la discapacidad asociada a la misma. Este estudio demostró que la calidad de vida, la funcionalidad del ámbito familiar y las actividades rutinarias diarias de los cuidadores familiares, se vieron afectadas como resultado del cuidado y sentimientos de preocupación permanente hacia el estado de salud del paciente. Así mismo, se observó que un $43 \%$ del total de la población de cuidadores evaluada, sufrió pérdidas significativas de oportunidades socio-laborales a lo largo de la vida por la enfermedad de su familiar (18).

Otro estudio realizado también en España, evaluó los índices de calidad de vida familiar, el apoyo social percibido y deterioro psicosocial en personas con discapacidad cognitiva, encontrándose relaciones estadísticamente significativas entre ambas variables. A mayor apoyo social percibido por la familia, mejor calidad de vida (19). En Chile se realizó un estudio que reveló los altos niveles de sobrecarga a los que están expuestos los cuidadores familiares, encontrándose que más de la mitad presenta sobrecarga intensa; así mismo, esta fue asociada con alteraciones del estado de salud general, calidad de vida y bienestar debido al estrés de no sentirse capaz de afrontar la situación y al gran esfuerzo físico que debían realizar para cumplir con las tareas de cuidado (20). 
En Colombia, los resultados de una investigación realizada en 36 familias de niños con diversos tipos de discapacidad evidenciaron una relación significativa entre el tipo de discapacidad y la calidad de vida familiar; siendo el dominio de funcionamiento psicológico el más afectado en la madre (21). Otro estudio realizado en la ciudad de Cali, demostró que la calidad de vida de las familias de personas adultas con discapacidad está afectada por la falta de oportunidades para la inclusión social que tienen estas personas, ocasionando efectos negativos sobre el bienestar tanto del individuo como de su familia (22).

La variable de calidad de vida es importante entenderla y contextualizarla hacia el tema de la discapacidad, puesto que es evidente que las familias de las personas con discapacidad se diferencian en sus características de aquellas que no tienen esta condición dentro de su núcleo, requiriendo, por tanto, atención psicosocial y orientación profesional integral para lograr favorecer el desarrollo de la persona con discapacidad y por ende de su familia. En este sentido, la calidad de vida de la familia se asocia al bienestar personal, el cual va más allá de lo individual, alcanzando así a la comunidad. Además, teniendo en cuenta que las personas con discapacidad tienen una participación social limitada, hay más elementos de riesgo que pueden resultar como amenazas para su calidad de vida y por tanto de su bienestar (23).

\section{APOYO SOCIAL PERCIBIDO EN FAMILIAS DE PERSONAS CON DISCAPACIDAD}

El concepto de apoyo o soporte social se origina en los años 70 como una variable primordial en estudios del área de la salud, especialmente en el afrontamiento del estrés en situaciones de crisis. Algunas definiciones dadas por expertos lo definen en términos de lazos perdurables que desempeñan un papel importante en la integración psicológica y física de 
una persona. Así mismo, se ha relacionado con factores existentes en el ambiente y que promueven el curso favorable de la enfermedad. Algunos autores exponen que los individuos se encuentran dentro de una red de relaciones sociales y estas se vuelven importantes cuando proveen el apoyo necesario en situaciones de crisis en la vida en general. Por tanto, el apoyo social debe entenderse como un concepto de muchos componentes, un conjunto de diferentes significados y explicaciones (24).

El apoyo social puede ser conceptualizado también a partir de diversas perspectivas, una cuantitativa-estructural, que evalúa la cantidad de vínculos o relaciones que establece el individuo o familia con su red social, que correspondería al apoyo social recibido y otra cualitativa-funcional que se centra en la existencia de relaciones significativas y la evaluación o apreciación subjetiva que realiza el individuo o familia de la adecuación del apoyo que recibe, esta correspondería al apoyo social percibido (25). El apoyo social percibido por una familia con la presencia de discapacidad, está relacionado con las características y capacidades de los miembros de la familia, con sus recursos disponibles, y las redes que tengan disponibles para satisfacer sus demandas durante la enfermedad, promover la capacidad de recuperación, y facilitar los procesos de adaptación (26).

Este también puede ser entendido como "la percepción hipotética que tiene una persona sobre los recursos sociales disponibles para ser asesorada y ayudada en caso de necesitarlo" (27); está relacionada con una dimensión de carácter subjetivo que se establece a partir de la percepción que tiene la familia cuidadora de la persona con discapacidad, de contar con la ayuda necesaria en caso de necesitarla, tanto para realizar actividades de la vida cotidiana como para resolver situaciones de emergencia. De igual forma, el apoyo social puede entenderse como aquel que se desprende de una red social, la cual puede estar relacionada con contactos sociales significativos, con la posibilidad de tener personas pares a las cuales expresarles los 
sentimientos más íntimos, o también como el apoyo perceptible o no que puede ser dado por los otros, o inferido por su presencia y que tiene efectos conductuales y emocionales beneficiosos en el receptor (27).

Existen numerosos estudios que demuestran el impacto que ejerce el apoyo social sobre los procesos relacionados con la salud y la enfermedad, así como su efecto beneficioso sobre la evolución de enfermedades como la depresión, enfermedades crónicas no transmisibles y discapacidad. Este es considerado un elemento protector de la salud en la familia de personas con discapacidad, puesto que modera los efectos negativos del estrés y contribuye así al bienestar y satisfacción con la vida $(25,26)$.

En este sentido, el apoyo social debe ser visto como un proceso complejo que implica una interacción entre las estructuras sociales, las relaciones sociales y los atributos personales y colectivos de la familia. También hace referencia a la disponibilidad del apoyo social en caso de ser requerido. Es considerado como moderador de estrés y como variable de gran relevancia en los procesos de salud-enfermedad que favorecerá los procesos de adaptación personal, familiar, escolar y social (28). Hay evidencias que, a mayor apoyo social, mayor estabilidad sintomatológica, por eso resulta fundamental proveer apoyos específicos a través de programas, en especial para las personas que viven en entornos con redes sociales pequeñas y poco diversas (28).

\section{MODELOS DE INTERVENCIÓN EN DISCAPACIDAD CENTRADO EN LA FAMILIA}

En la medida en que el proceso de discapacidad del miembro de la familia tiende a avanzar, y a volverse más complejo, se genera también un avance y una mayor tensión en la dinámica familiar, sobre todo cuando la percepción de esta es negativa con respecto a la recuperación y logro de mejores 
capacidades cognitivas y sociales del miembro que se encuentra en dicha situación. Si esto sucede, es probable que puedan surgir nuevas alteraciones de carácter psicopatológico en la persona con discapacidad, y que a su vez se vea afectada en mayor medida la familia al encontrarse sin los recursos físicos y emocionales necesarios y suficientes para hacerle frente a esta situación considerada como una problemática de gran complejidad (11).

Es ante estas situaciones, que resulta fundamental para la familia, contar con el apoyo de un equipo profesional interdisciplinario, de tal forma que pueda recibir todos los conocimientos necesarios sobre la condición de salud real del miembro con discapacidad, donde la identificación de habilidades y oportunidades para recuperar el equilibrio familiar resultan fundamentales. En este proceso, el reconocimiento y aceptación por parte de la familia de la nueva condición de salud del miembro familiar, permitirá mejorar tanto la calidad de vida de este, como los niveles de funcionalidad y dinámica familiar. Así mismo, esta comprensión y aceptación por parte de la familia, le permitirá una mejor adaptación al entorno social en el que normalmente se desenvuelven (11).

Los modelos de intervención para personas con discapacidad centrados en la familia, tienen como objetivos principales mejorar y mantener la calidad de vida tanto de la persona que padece la discapacidad como la de su grupo familiar. Para esto, es necesario conocer la percepción que tiene la familia sobre sus prioridades y los factores que consideran podrían ser relevantes para lograr un mejor equilibrio y una mejor funcionalidad, aspecto que repercutirá de manera importante en dicho propósito. Bajo este enfoque, el equipo de profesionales se centrará en el contexto familiar como un escenario de interacción, espacio de confluencia y apoyo entre sus miembros; así mismo, se pondrá un especial énfasis en la identificación de las barreras y obstáculos especialmente en los procesos de comunicación y se identi- 
ficarán las estrategias para eliminarlas, tomando como base la aceptación del hecho de que cada persona que conforma el grupo familiar hace parte de una red natural de apoyo, y de esta forma, actuar en consecuencia (15).

Las principales ventajas que posee el enfoque de intervención centrado en la familia, en comparación con los métodos de intervención a nivel individual, son de diverso orden. Principalmente se resalta la consideración del enfoque sistémico de la familia como la unidad principal de intervención, $\mathrm{y}$, por tanto, el reconocimiento de sus necesidades y el valor que le da a la interdependencia entre cada uno de los miembros; por otro lado, este sistema da un gran valor a los vínculos positivos que existen en la familia, los fortalece y busca el equilibrio entre cada individuo y la familia en su totalidad.

En síntesis, las principales características del enfoque de intervención centrado en la familia son: respeto por los valores, creencias y metas de la unidad familiar; sensibilización y empoderamiento de la familia para la toma de decisiones, fortalecimiento de la resiliencia familiar, y trabajo interdisciplinario coordinado con la familia (29).

\section{CONCLUSIONES}

En síntesis, resulta fundamental reconocer la importancia que tiene la familia dentro de los procesos de rehabilitación integral de las personas con discapacidad, y más aún en su inclusión a la vida en sociedad. Esto queda claro al comprender que la familia es el núcleo básico, natural y primer escenario de socialización con el que cuenta la persona con discapacidad para su desarrollo; es en la familia donde este encuentra la posibilidad de evolucionar y desarrollar al máximo todas sus potencialidades internas (30). 
De igual forma, es importante destacar, que dentro de las principales características de la familia como sistema complejo abierto, se encuentra su capacidad de cambio y reestructuración ante las dificultades y obstáculos que la vida va generando. Un ejemplo de esto es la capacidad que la familia puede desarrollar para asumir conscientemente las circunstancias actuales familiares, la aceptación y toma de decisiones para la distribución equitativa de las responsabilidades, sobre todo, de las relacionadas específicamente con las necesidades de la persona que presenta la discapacidad (30).

Sin embargo, queda claro que para lograr estas nuevas adaptaciones, y evolucionar, la familia necesita del apoyo y la asesoría del equipo de profesionales que implementan los procesos de Atención Primaria en Salud, para quienes, el abordaje de la familia constituye y debe constituir un aspecto de gran relevancia y todas las actividades desarrolladas bajo esta orientación coadyuvarán al logro de la autonomía e inclusión en la vida en sociedad de las personas con discapacidad y sus familias. Se destaca que este abordaje debe estar orientado en doble vía, a través de la interacción entre profesionales y familia, de tal forma que puedan generarse mejores canales de comunicación y resolución efectiva de conflictos y desequilibrios de la propia funcionalidad familiar, la cual se verá reflejada en el estado de salud general del miembro que presenta la discapacidad (30).

El trabajo integrado entre profesionales y familia permite el desarrollo de oportunidades de aprendizajes significativos, creativos y eficaces para ambos, basados en el reconocimiento de las familias sobre sus derechos y deberes, donde la meta principal es lograr un desarrollo integral y armónico de la familia en relación con la sociedad (30). El objetivo, será entonces formular de manera conjunta y cooperativa estrategias de intervención integrales para la inclusión social de las personas con discapacidad y sus familias, de tal forma que se puedan generar redes de apoyo social donde 
se integren también otras organizaciones gubernamentales y no gubernamentales, en la búsqueda de un objetivo común: la equiparación de oportunidades y el logro de calidad de vida familiar.

Se concluye también la importancia que tiene introducir el concepto de calidad de vida en el contexto de una familia que tiene uno de sus miembros con discapacidad. Este nos insta necesariamente a ubicarnos en el nuevo paradigma del concepto de discapacidad, un concepto articulado a la capacidad de interacción con el medio, con lo social, y no necesariamente ligado a una deficiencia corporal (31).

Este enfoque, considerado de carácter psicosocial, promueve la protección permanente de los derechos de las personas con discapacidad en la búsqueda de equiparación de oportunidades. Por tanto, la discapacidad pasa de ser un fenómeno estrictamente biológico e incapacitante, a considerarse un fenómeno social, el cual está ligado a las posibilidades que pueda brindar la sociedad para la eliminación de barreras físicas y humanas, y que por tanto sea posible la integración de la población con discapacidad. Esta nueva visión favorece la visibilización de las capacidades personales y sociales de las personas con discapacidad y el reconocimiento de estas, como sujetos activos de la sociedad, capaces de aportar elementos importantes para la planificación de programas y servicios dirigidos hacia ellos mismos (32).

Otro aspecto fundamental es la comprensión que pueda tener todo el equipo humano que interactúa con la persona con discapacidad sobre el papel que desempeña la familia como el eje central para lograr la articulación de la persona con discapacidad a la sociedad (33). Por tanto, su participación y retroalimentación en la programación de intervenciones multidisciplinares es prioritaria para la generación de procesos de cambio reales y perdurables en el tiempo (34). Estas intervenciones deberán incluir necesariamen- 
te estrategias de conciliación y corresponsabilidad familiar que permitan garantizar el desarrollo integral tanto de la persona con discapacidad como de la propia familia.

Sin embargo, todas las disposiciones de programas y servicios basados en lo anterior, tendrían un efecto e impacto limitado si no se realizan oportunamente jornadas de sensibilización dirigidas a toda la población en general, de tal forma que se pueda prevenir el desarrollo de acciones negativas, principalmente de discriminación y exclusión social que puedan vulnerar los derechos de las personas con discapacidad (9).

Las familias tienen una gran necesidad de recibir apoyo y acciones dirigidas a la comunidad en general para la creación de conciencia social en torno a la inclusión de las personas con discapacidad. Está claro que aún falta mucho por hacer en este aspecto; es una realidad que la sociedad actualmente no está empoderada sobre la importancia de este asunto y la realidad que encontramos no es solo de falta de apoyo sino también de generación de situaciones adversas que bloquean el libre desarrollo de la vida de las personas con discapacidad y sus familias. De igual forma, es evidente la deficiencia que existe en las instituciones educativas en relación con este tema en los procesos de formación de los profesionales, sobre todo, los que se encargarán en un futuro de los servicios sociales, educativos y sanitarios de las personas con discapacidad y sus familias (15).

A través del tiempo, y con el desarrollo de avances sociales, educativos y científicos, las familias de personas con discapacidad han comenzado a ocupar un lugar central en la sociedad, desplazando las miradas hacia ellas y hacia la implementación de servicios de atención integrales que promuevan su bienestar, esto las ha llevado a reflejarse más como familias resilientes, proceso que favorece tanto la recuperación y mantenimiento de la calidad de vida familiar como el fortalecimiento de un nuevo modelo 
de calidad de vida donde el papel central no lo ocupa necesariamente la persona que padece la discapacidad con sus deficiencias y necesidades, sino también la familia, con sus necesidades, fortalezas y experiencias positivas. Esta es la base para la eficacia de las intervenciones dirigidas a la población con discapacidad en busca de la inclusión social y equiparación de oportunidades, un enfoque de intervención integral sistémico, centrado en la familia (29).

Este enfoque permite el diseño, la implementación y evaluación efectiva de programas y proyectos basados en la colaboración y trabajo intersectorial para la transformación de los roles asumidos tradicionalmente por las familias, por los profesionales y la sociedad en general, facilitando el restablecimiento del equilibrio familiar, y de la relación entre profesionales y familia. Este nuevo enfoque es reconocido como el enfoque centrado en la familia, el cual promete grandes cambios positivos en materia de derechos e inclusión social de las personas con discapacidad y sus familias $(29,35)$.

Por último, al ser la familia la primera y más importante red de apoyo para la persona en general y especialmente para la que presenta discapacidad, resultan fundamentales los procesos de investigación tanto observacionales como de intervención que puedan realizarse para obtener elementos diagnósticos suficientes que permitan conocer las necesidades reales de estas familias, y para definir de la manera más acertada intervenciones específicas para mejorar la calidad de vida y funcionalidad familiar, asumiendo que el apoyo social juega un papel fundamental en el mantenimiento de la salud de los individuos y cuidadores al facilitar diversas conductas adaptativas en situaciones de estrés (27). Este acercamiento teórico nos sirve de guía para los objetivos aquí planteados por los investigadores y establece, además, una línea de base para avanzar en el proceso de investigación de este tema tan fundamental para una sociedad incluyente. 


\section{REFERENCIAS BIBLIOGRÁFICAS}

1. Araya S. De lo invisible a lo cotidiano. Familias y discapacidad. Rev. Electrónica Actualidades investigativas en educación. 2007; 7(3):1-21.

2. Pagano L. Las personas con discapacidad y sus familias bajo el prisma de la Convención sobre los Derechos de las personas con discapacidad. Rev. del Instituto de Ciencias Jurídicas de Puebla. 2010: 4(16):79-96.

3. Verdugo M, Rodríguez A. Guía de Intervención y Apoyo a Familias de Personas con Discapacidad. Instituto Universitario de Integración en la Comunidad. Universidad de Salamanca.

4. Meler I. Las familias. Subjetividad y Procesos Cognitivos. 2008; 12:158-188.

5. Góngora G. Apoyo social en familias monoparentales y nucleares con hijos con discapacidad: un estudio comparativo. Rev. Col. Med. Fis. Rehab. 2013; 23(2):141-146.

6. Sánchez P. Primera infancia y discapacidad en Chile: Revisión y enfoque actual a los programas de gobierno. Rev. Med. Clin. Condes. 2015; 26(4):520-526.

7. Silva T. La familia de la persona con discapacidad mental: una intervención desde Trabajo Social. Interacción y Perspectiva. Revista de Trabajo Social. 2015; 5(1):113-129.

8. Salcedo R, Liébana J, Pareja J, Real S. Las consecuencias de la discapacidad en familias de la ciudad de Ceuta. Revista Española de Orientación y Psicopedagogía. 2012; 23(2):139-153.

9. Mercado E, Aizpurúa E, García L. Características, percepciones y necesidades sociales de los niños y niñas con discapacidad y sus familias. Portularia. 2012; 12(2):69-80.

10. Yuan Chen J, Clark M. Family Function in Families of Children with Duchene Muscular Dystrophy. Fam Community Health. 2007; 30(4):296-304. 
11. Rea A, Ampudia A, García M. Caracterización de los conocimientos de las madres sobre la discapacidad de sus hijos y su vínculo con la dinámica familiar Acta Colombiana de Psicología. 2014; 17(1):91-103.

12. Córdoba L, Gómez J, Verdugo M. Calidad de vida familiar en personas con discapacidad: Un análisis comparativo. Univ. Psychol. 2008; 7(2):369-383.

13. Rodríguez M, Guerrero C. La familia como promotora de la independencia en niños y niñas con discapacidad motora. Revista Electrónica Educare. 2012. 16(1):99-111.

14. Peralta F, Arellano A. Familia y discapacidad. Una perspectiva teórico-aplicada del enfoque centrado de la familia para promover la autodeterminación. Education and Psychology. 2010; 22(8):1339-1362.

15. González A, Simón C, Cagigal V, Blas E. La calidad de vida de las familias de personas con discapacidad intelectual. Un estudio cualitativo realizado en la comunidad de Madrid. Revista Española de Orientación y Psicopedagogía. 2013; 24(1):93-109.

16. Córdoba L, Verdugo M, Gómez J. Escala de calidad de vida familiar para familias de personas con discapacidad. Adaptación para Colombia. Madrid, España. 2011.

17. Achury D, Castaño H, Gómez L, Guevara N. Calidad de vida de los cuidadores de pacientes con enfermedades crónicas con dependencia. Investigación en Enfermería: imagen y desarrollo. 2011; 13(1):27-46.

18. Martínez A, Nadal S, Beperet M, Mendioróz P. Sobrecarga de los cuidadores familiares de pacientes con esquizofrenia: factores determinantes. Anales Sis San Navarra. 2000; 23(1):101-110.

19. Navarro D, García S, Carrasco O, Casas A. Calidad de vida, apoyo social y deterioro en una muestra de personas con trastorno mental grave. Intervención psicosocial. 2008; 17(3):321-336.

20. Flores E, Rivas E, Seguel F. Nivel de sobrecarga en el desempeño del rol del cuidador familiar de adulto mayor con dependencia severa. Ciencia y Enfermería. 2012; 18(1):29-41. 
21. Ballesteros B, Novoa M, Muñoz L, Suárez F, Zarante I. Calidad de vida en familias con niños menores de dos años afectados por malformaciones congénitas. Perspectiva del cuidador principal. Univ. Psychol. 2006; 5(3):457-473.

22. Córdoba L, Mora A, Bedoya A. Familias de adultos con discapacidad intelectual en Cali, Colombia, desde el modelo de calidad de vida. Psykhe; 2007; 16(2):29-42.

23. Muñoz Y, Poblete Y, Jimenez A. Calidad de vida familiar y bienestar subjetivo en jóvenes con discapacidad intelectual de un establecimiento con educación especial y laboral de la ciudad de Talca. Interdisciplinaria. 2012; 29(2):207-221.

24. Angarita OM, González E. Apoyo social: Elemento clave en el afrontamiento de la enfermedad crónica. Enfermería Global. 2009; 16:1-11.

25. Vivaldi F, Barra E. Bienestar psicológico, apoyo social percibido y percepción de salud en adultos mayores. Terapia psicológica. 2012; 30(2):23-29.

26. Castillo J, Muñoz B, Moruno M. Validación del cuestionario MOS de apoyo social en Atención Primaria. Medicina de Familia. 2005; 6(1):10-18.

27. Vega A, Olga M. Percepción del apoyo social funcional en cuidadores familiares de enfermos crónicos. 2011. Disponible en: http://site. ebrary. com. dibpxy.uaa.mx/lib/univeraguascalientessp/docDetail.ac

28. Navarro D, García S, Delgado H, Carrasco O, Casas A. Calidad de vida, apoyo social y deterioro en una muestra de personas con trastorno mental grave. Intervención Psicosocial. 2008; 17(3):321-336.

29. Arellano A, Peralta F. El enfoque centrado en la familia, en el campo de la discapacidad intelectual ¿Cómo perciben los padres su relación con los profesionales? Revista de Investigación Educativa. 2015; 33(1):119-132.

30. El abordaje de la discapacidad desde la atención primaria en salud. 
Universidad Nacional de Córdoba. Organización Panamericana de la Salud. Argentina. 2008.

31. Córdoba L, Soto G. Familia y discapacidad: intervención en crisis desde el modelo ecológico. Psicología Conductual. 2007; 15(3):525-541.

32. Suriá R. Discapacidad y empoderamiento: análisis de esta potencialidad en función de la tipología y etapa en la que se adquiere la discapacidad. Anuario de Psicología. 2013; 43(3):297-311.

33. Valls M, Vila M, Pallisera M. La inserción de las personas con discapacidad en el trabajo ordinario. El papel de la familia. Revista de Educación. 2004; 334:99-117.

34. Marín M. Conversaciones entre la familia y el equipo terapéutico en la discapacidad adquirida. Pensamiento psicológico. 2008; 4(11):151-166.

35. Barrientos P. La participación familia-escuela en México desde un enfoque inclusivo: reflexiones sobre familia y discapacidad. Revista Latinoamericana de Educación Inclusiva. 2013; 7(2):175-193.

\footnotetext{
Cómo citar este capítulo:

García Puello F, Crissién Quiroz E. (2018). Familia y discapacidad: calidad de vida y apoyo social percibido. In Pinillos Patiño Y, Herazo Beltrán Y, Vidarte Claros JA, Crissién Quiroz EM, Suárez Palacio D, García Puello F, et al. Caracterización de la discapacidad en el distrito de Barranquilla. Una mirada conceptual y experiencial. Barranquilla: Universidad Simón Bolívar, p. 179-205.
} 